
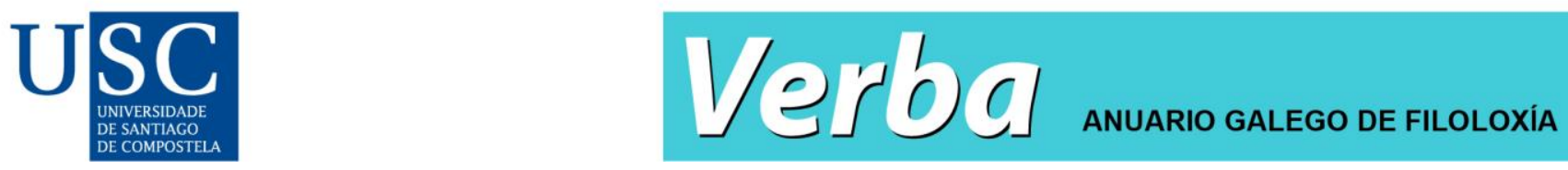

Verba: Anuario Galego de Filoloxía, 48, 2021. ISSN: 2174-4017

https://doi.org/10.15304/verba.48.7089

Revista de libros

\title{
Antonio Fábregas (2020): Las categorías funcionales. Madrid: Síntesis, 267 pp.
}

\author{
Elena Felíu Arquiola ${ }^{1}$ \\ 1Universidad de Jaén, España
}

Desde finales de los años 80, los principales avances en sintaxis dentro del marco teórico de la Gramática Generativa han surgido del estudio detallado de las categorías funcionales, tema principal del libro que aquí reseñamos. Tal como indica en el prólogo su autor, Antonio Fábregas, las cuestiones que podrían abordarse en relación con las categorías funcionales exceden con mucho la extensión de un manual de estas características, por lo que resulta necesario realizar una selección de los contenidos. En este sentido, el autor aclara que se va a centrar fundamentalmente en la sintaxis de las categorías funcionales, así como —en menor medida- en su semántica y en su adquisición y pérdida, todo ello dentro de una orientación generativista.

Las categorías funcionales se divide en dos partes. La primera consta de cuatro capítulos y se centra en cuestiones de tipo general, mientras que la segunda contiene ocho capítulos y aborda de forma detallada la estructura funcional básica de las oraciones y de los principales constituyentes sintácticos.

Como hemos anticipado, la primera parte del libro, titulada "Cuestiones básicas sobre las categorías funcionales", engloba los capítulos 1 al 4. En el primero de ellos, cuyo título es "La noción de categoría funcional", se define este concepto y se compara con el de categoría léxica a partir de una serie de criterios habitualmente empleados en la bibliografía, como la diferenciación entre clases cerradas y clases abiertas; la mayor o menor independencia fonológica y morfológica de cada tipo de categoría; la mayor o menor rigidez en la selección gramatical que cada una de estas categorías realiza; su tipo de significado — procedimental frente a léxico-; finalmente, su incapacidad o su capacidad de definir relaciones argumentales, cuestión polémica que se retoma más adelante (cap. 8). En este primer capítulo también se presenta una interesante discusión sobre dos ejemplos concretos de clases de unidades cuya tipificación como categoría funcional o categoría léxica resulta problemática, discusión que pone de manifiesto que esta distinción admite grados: por una parte, las adposiciones (preposiciones y posposiciones) y algunas conjunciones; por otra, los verbos auxiliares (está durmiendo), los verbos ligeros (dar pena) y los sustantivos ligeros (un par de días, una taza de té). Finalmente se aborda el vínculo existente entre categorías léxicas y funcionales según la propuesta de Grimshaw (1991): las categorías funcionales se 
ordenan de forma rígida y se asocian a categorías léxicas específicas, de las que forman una proyección extendida.

En el capítulo 2 se realiza un breve recorrido histórico por la evolución del tratamiento de las categorías funcionales en el generativismo, desde las reglas de reescritura, pasando por la teoría de la X barra hasta llegar a propuestas más recientes (Chomsky 1995). Posteriormente, se argumenta el papel básico de las categorías funcionales en la configuración de la estructura sintáctica: la concordancia, el caso y el movimiento. Así, se vincula la concordancia entre el verbo y el constituyente que realiza la función sintáctica de sujeto con la categoría funcional T (Tiempo), que define el concepto de oración. Esta concordancia, que implica a los rasgos phi (persona, número y género), se concibe en sintaxis generativa como una operación de valoración o especificación de rasgos no especificados entre una meta (rasgos con valor, interpretables) y una sonda (rasgos sin valor, no interpretables), de tal forma que se establece "una relación por la cual los rasgos sin valor de la sonda copian la información que contienen los rasgos ya valorados de la meta" (p. 54). En cuanto al caso, se muestra la relación entre concordancia y asignación de caso. Igualmente, se presenta la distinción entre caso estructural -vinculado con categorías funcionales - y caso inherente y se muestra cómo progresivamente la teoría sintáctica ha ido relacionando también este segundo tipo con proyecciones funcionales. Finalmente, en relación con el movimiento, se distingue el movimiento argumental o movimiento-A y el movimiento no argumental o movimiento-A'. Mientras que el primero se relaciona con la necesidad de un constituyente de recibir función sintáctica y se da en el interior de una oración finita, el segundo está motivado por otro tipo de relaciones como la modalidad y puede atravesar oraciones. Ambos tipos tienen en común el hecho de que los elementos desplazados se mueven a proyecciones funcionales, así como el hecho de que la causa del movimiento sea satisfacer alguna propiedad del elemento desplazado y de la proyección funcional a la que se mueve mediante la valoración de rasgos.

El capítulo 3 se titula "Adquisición y pérdida de las categorías funcionales". Sus dos primeros apartados están dedicados al papel de las categorías funcionales en la adquisición de la L1 y de la L2, respectivamente. Así, en relación con la adquisición de la L1 desde la perspectiva de las teorías innatistas, se contrasta la hipótesis continuista — según la cual el punto de partida para adquirir una lengua es una estructura sintáctica completa, que incluye categorías léxicas y funcionales- y la hipótesis maduracional — según la cual en la adquisición de la L1 se parte de una plantilla o estructura sintáctica incompleta, en la que faltan las categorías funcionales-. En lo que respecta a la adquisición de una L2, se discute el papel de las categorías funcionales en relación con los fenómenos de transferencia y de acceso. El tercer apartado se centra en la aparición de categorías funcionales en los procesos históricos de gramaticalización. Finalmente, los dos últimos apartados de este capítulo abordan la pérdida de categorías funcionales en dos sentidos distintos: por una parte, en los procesos de atrición o erosión de la L1; por otra, en las patologías del lenguaje, tanto en las patologías del desarrollo como en las patologías adquiridas. En concreto, en este último apartado, se aportan pruebas vinculadas con los distintos tipos de patologías para apoyar la distinción entre categorías léxicas y funcionales.

La primera parte del libro se cierra con el capítulo 4, titulado "Cinco preguntas sobre las categorías funcionales", en el que, mediante la formulación de cinco preguntas que dan título a cada uno de los apartados, se plantea una serie de cuestiones conflictivas que implican a las categorías funcionales en la teoría sintáctica actual dentro del marco generativista, al tiempo que 
se anticipan contenidos que se abordarán en la segunda parte del manual. Así, como respuesta a la pregunta “¿Hasta dónde llegan las atribuciones de las categorías funcionales?”, se presenta la idea de que las categorías funcionales pueden intervenir en la definición de las funciones informativas (cap. 7), de los papeles temáticos (cap. 8) y de las categorías gramaticales en sí mismas, así como de nociones tradicionalmente vinculadas a las categorías léxicas (contable vs. no contable, individual vs. colectivo). En segundo lugar, la pregunta de $i$ “Cómo de detallada es la estructura funcional?" permite al autor introducir los dos principales modelos sintácticos en el marco del generativismo en la actualidad, que irá contrastando en el resto del libro: los modelos cartográficos, que proponen una estructura funcional muy detallada, como es el caso de Cinque (1999), frente a los modelos minimistas (Chomsly 1995), caracterizados por la "parsimonia representacional", esto es, por "la hipótesis de que las lenguas humanas construyen las estructuras partiendo de una cantidad muy reducida de primitivos sintácticos, con muy pocas proyecciones funcionales" (p. 113). Fábregas anticipa que en la segunda parte del manual se decantará por una postura intermedia, más próxima al minimismo, aunque aludirá a la cartografía al hablar de funciones informativas y de complementos no obligatorios. La tercera pregunta (" ¿Son universales las categorías funcionales?") permite al autor exponer tres puntos de vista distintos: la propuesta de que las categorías funcionales no son necesariamente universales, como se considera en tipología lingüística; la propuesta de que dichas categorías son siempre universales y lo que varía en las lenguas particulares es su manifestación superficial, como en el generativismo clásico; finalmente, una propuesta intermedia muy interesante que consistiría en considerar que las categorías funcionales son universales, pero serían pocas y muy generales, y de una lengua a otra coincidirían solo en su función en la oración (por ejemplo, anclar un predicado a una situación), mientras que podrían variar en relación con el tipo de concepto que gramaticalizan (por ejemplo, tiempo o espacio) (Wiltschko 2014). La cuarta pregunta (“ ¿Hay paralelismos entre distintas categorías funcionales?") plantea la posible existencia de paralelismos entre la estructura funcional de la oración y la estructura funcional del sintagma nominal. Finalmente, en el último apartado, titulado “¿Son las categorías funcionales responsables de la variación sintáctica?”, el autor presenta la idea dominante en gramática generativa según la cual la variación gramatical — tanto intralingüística como interlingüística - se relaciona con una distinta especificación de las categorías funcionales y muestra distintas posibilidades.

Bajo el título "La estructura funcional de las principales categorías", la segunda parte del libro se centra en la estructura funcional básica de las oraciones y de otros constituyentes sintácticos. En el capítulo 5 ("Principales categorías funcionales de la cláusula (1): tiempo, aspecto, modo y polaridad") se argumenta a favor de considerar ST como núcleo de la oración, pues la información contenida en ese núcleo define el significado básico de la oración (localiza un predicado en el tiempo y permite que la proposición denote un valor de verdad) y su distribución. Igualmente se argumenta a favor de las categorías funcionales SAsp (Aspecto), SModo y SPol (Polaridad) y se propone la siguiente estructura funcional: [SPol [ST [SAsp [SV]]]]]. En cuanto al modo, se defiende la idea de que el modo deóntico y el modo epistémico se vinculan con categorías funcionales diferentes, ubicadas en distintas posiciones en la estructura: [SModo epist [ST [SAsp [SMododeon [SV]]]]]. El resto del capítulo (apartados 5.2, 5.3 y 5.4) se dedica a la exposición detallada de la semántica y la sintaxis de estas tres categorías funcionales (SAsp, SModo, SPol).

En el capítulo 6, titulado "Principales categorías funcionales de la cláusula (2): modalidad, evidencialidad y relaciones interoracionales", se argumenta la necesidad de proponer una categoría funcional vinculada con la modalidad oracional situada por encima de ST. Se trata de SC 
(Complementante), cuya función es conectar la oración con el contexto. El SC es responsable de suspender el valor de verdad de las proposiciones en oraciones interrogativas, exclamativas e imperativas, entre otras. De este modo, C es un núcleo funcional que convierte la proposición en un enunciado que codifica, entre otras cosas, la intención con que se emite. Posteriormente se aborda la noción de evidencialidad, entendida como "gramaticalización en la estructura lingüística del origen del que procede la evidencia que tiene un hablante para presentar como verdadero un estado de cosas" (p. 169), y se discuten dos propuestas: por una parte, aquella según la cual la evidencialidad se expresa mediante la categoría funcional C; por otra parte, una segunda opción, desarrollada en el seno de la cartografía, que defiende una categoría funcional SEvid, distinta de SC, aunque sintácticamente próxima. A continuación, se caracteriza el papel que desempeña la categoría funcional $\mathrm{C}$ en el establecimiento de relaciones interoracionales, concretamente, en la subordinación. En este sentido, C pasa a definirse de forma menos específica como una categoría que conecta la oración con el contexto extraoracional, incluyendo así tanto el contexto extralingüístico como el lingüístico. Desde la perspectiva de su papel en la subordinación, C es un núcleo que sirve para adaptar una proposición de manera que pueda usarse como miembro de otra proposición. El capítulo termina con una breve discusión de la propuesta de que C puede desempeñar un papel también en la coordinación y quizá en otras relaciones interoracionales como la correlación y la interordinación, discusión que el autor zanja de forma algo apresurada por motivos de espacio y que sin duda merecería una mayor atención.

Bajo el título "Categorías funcionales y funciones informativas", el capítulo 7 aborda la relación entre las funciones informativas y SC, capa funcional que, como se vio en el capítulo anterior, se ocupa del contexto. En el primer apartado, Fábregas expone algunos conceptos fundamentales relacionados con la estructura informativa de la oración, tales como tópico (de familiaridad, contrastivo, de cambio de tema) y comentario, foco (informativo, contrastivo, mirativo) y presuposición, así como la noción de Transfondo Compartido. Cabe señalar que, a lo largo del capítulo, este último concepto recibe también las denominaciones de Transfondo Comunicativo (p. 183) y Transfondo Común (p. 184), lo que podría resultar confuso. En los dos últimos apartados, el autor discute dos aproximaciones teóricas a la representación sintáctica de estructura informativa. Por una parte, presenta la propuesta cartográfica, que descompone $\mathrm{C}$ en varias proyecciones funcionales: SFuerza (que define la modalidad); STop (Tópico) y SFoc (Foco), que definen la estructura informativa, y SSub (Subordinación), cuya función es adaptar la proposición para que pueda funcionar dentro de otra estructura. Por otra, presenta la propuesta minimista, en la que se analizan los mismos hechos mediante un solo SC con varios especificadores.

En el capítulo 8, titulado "Categorías funcionales y estructura argumental", Fábregas presenta argumentos a favor de transferir la definición de la estructura argumental, al menos parcialmente, a las categorías funcionales, mientras que en las teorías clásicas de sintaxis generativista la estructura argumental venía definida por las categorías léxicas. En este sentido, el autor presenta las proyecciones habitualmente reconocidas en la bibliografía. Por un lado, expone la propuesta de Larson (1988) de descomponer SV en dos proyecciones: Sv, relacionada con el inicio y la causa del cambio (definiría al agente), y SV, relacionado con el cambio en sí y su resultado (definiría al argumento interno). Presenta también desarrollos de esta propuesta, como los de Harley (1995) y Ramchand (2008). Por otro lado, caracteriza la categoría funcional SApl (Aplicativo), que se encarga de introducir un argumento marcado como dativo. Finalmente, en relación con los complementos no obligatorios o adjuntos, el autor discute la propuesta estándar de considerarlos modificadores no obligatorios que se añaden a las proyecciones funcionales, así 
como la propuesta cartográfica según la cual los adjuntos son tratados como especificadores de categorías funcionales especializadas en designar distintas nociones (Manera, Frecuencia, Velocidad, etc.).

Tras cuatro capítulos dedicados a la estructura funcional de la cláusula y del sintagma verbal, el capítulo 9 se centra en las categorías funcionales del sintagma nominal ("Referencialidad y cuantificación: la estructura funcional del SN"). Fábregas presenta argumentos a favor de al menos tres proyecciones funcionales por encima de SN: SD (Determinante), SCu (Cuantificador) y SNum (Número). La categoría funcional D codifica nociones relacionadas con la referencialidad, tales como la definitud, la especificidad y la deíxis. Por su parte, la categoría funcional $\mathrm{Cu}$ se relaciona con la cuantificación. Tras explicar brevemente que los cuantificadores se conciben como operadores - esto es, expresiones que controlan la interpretación de una variable que se encuentra en la misma estructura-, el autor expone que se suelen distinguir dos posiciones sintácticas para estos elementos incluso en teorías no cartográficas: los cuantificadores universales se situarían por encima de SD, mientras que los existenciales se ubicarían por debajo de SD. Finalmente, el autor indica que se reconoce al menos una proyección funcional vinculada con los accidentes gramaticales del SN, el SNum, y aporta argumentos que muestran la diferente naturaleza del género, que sería un rasgo del nombre. En suma, la estructura funcional propuesta para el SN sería la siguiente:

\section{$\left[\begin{array}{llllllll}\mathrm{SCu} & \mathrm{Cu}^{1} & {[\mathrm{SD}} & \mathrm{D} & {[\mathrm{SCu}} & \mathrm{Cu}^{2} & {[\mathrm{SNum}} & [\mathrm{SN}]]]\end{array}\right]$}

La estructura funcional del sintagma adjetivo se aborda en el capítulo 10, titulado "Gradación y predicación: la estructura del SA". Fábregas restringe su objeto de estudio a los adjetivos calificativos graduables, de forma que no trata ni los adjetivos relacionales ni los adverbiales. En relación con los adjetivos calificativos graduables, el autor diferencia dos proyecciones funcionales y presenta argumentos que justifican esta propuesta: por una parte, SPred sería la proyección responsable de establecer una predicación; por otra, SGrado sería la proyección relacionada con el grado del adjetivo. El capítulo termina con una breve presentación de la semántica de las escalas, asociada a la capa léxica de A. Fábregas menciona brevemente la posibilidad de que las diferencias entre escalas acotadas y no acotadas puedan codificarse con una proyección funcional.

El capítulo 11, titulado "Pronombres, adposiciones y adverbios", se tratan brevemente las clases de palabras no abordadas hasta el momento en el libro. En relación con los pronombres, se presenta la idea de que, a diferencia de otras expresiones nominales, carecen de capa léxica que exprese un predicado, por lo que son proyecciones de D o $\mathrm{Cu}$. En cuanto a los pronombres personales, se presentan argumentos a favor de considerar la existencia de una proyección funcional SPer (Persona), vinculada con el rasgo gramatical de persona que caracteriza a este tipo de pronombres. En cuanto a las preposiciones, se retoma la diferencia apuntada en el capítulo 1 entre preposiciones léxicas y preposiciones funcionales y se presenta la posibilidad de descomponer el SP en una capa léxica (SP) y una capa funcional (Sp), de forma paralela a lo que se hizo en el caso del SV en el capítulo 8. En relación con los adverbios, se destaca la heterogeneidad de los elementos que se suelen adscribir a esta clase de palabras, que solo tienen en común entre ellos dos propiedades negativas: carecer de accidentes gramaticales y no poder usarse por sí mismos para relacionar oraciones u otras estructuras. Fábregas considera, acertadamente a nuestro entender, que la clase del adverbio no está definida correctamente en la tradición y que en realidad al menos algunos adverbios son manifestaciones de otras categorías ya examinadas. En 
concreto, el autor esboza brevemente la idea de que determinados adverbios, como los negativos y afirmativos, podrían ser núcleos de SPol, mientras que los adverbios de cantidad y de grado podrían ser manifestaciones de SCu y de SGrado, respectivamente. Otros adverbios, como los pronominales deícticos (aquí, ahora), podrían ser la manifestación de una categoría léxica con parte de su estructura funcional, en concreto, proyecciones de SD (Rodríguez Ramalle, 1995), mientras que muchos adverbios en -mente (rápidamente, curiosamente, francamente) podrían tratarse, según Fábregas, como estructuras funcionales adjetivales. Consideramos que esta exploración de la posibilidad de aplicar las categorías funcionales a la caracterización de ese cajón de sastre que, como ya señalara Bosque (2015 [1989]), constituye el adverbio puede resultar muy prometedora.

Finalmente, con el título de “Conclusión: más allá de las categorías funcionales”, el capítulo 12, de breve extensión, recoge las principales conclusiones y abre nuevos caminos. Así, Fábregas manifiesta su preferencia por las configuraciones sintácticas como medio de análisis de los fenómenos lingüísticos, en lugar de recurrir a la multiplicación de categorías funcionales, lo que lo aleja de las propuestas cartográficas. Las últimas reflexiones del autor buscan trazar vínculos entre las estructuras sintácticas y las propiedades generales de la cognición humana y presentar algunas propuestas recientes en esa línea, que considera prometedora.

Cada capítulo incluye unos "Ejercicios de profundización", en ocasiones divididos en dos niveles (intermedio y avanzado), que ayudan al lector a ahondar en determinados aspectos tratados en la exposición o a abordar cuestiones que no ha sido posible tratar en el cuerpo del capítulo, como sucede con la distinción entre adverbios léxicos y funcionales o con la adscripción de las interjecciones a uno u otro grupo de unidades (cap. 1). Aunque en el prólogo del libro se justifica el hecho de que no se proporcionen las soluciones, creemos que los lectores habrían resultado beneficiados si se hubieran incluido. Sin ser necesario ofrecer respuestas absolutas algo que el autor con buen tino quiere evitar-, sí podrían haberse proporcionado varias respuestas alternativas que funcionasen como posibles modelos o como sugerencias y que guiasen al lector en la elaboración de su propia respuesta.

Entre las numerosas virtudes de este manual se encuentran, por una parte, el hecho de que su lectura resulte amena, pese a la complejidad del tema tratado, lo que sin duda se debe al estilo dinámico y a la abundancia de ejemplos. Por otra parte, podemos destacar la sólida fundamentación de las distintas argumentaciones que se desarrollan, así como el hecho de que se ofrezca una bibliografía muy actualizada. Igualmente, el manual presenta los principales debates que se dan en la actualidad en teoría sintáctica en el marco del generativismo. También es de agradecer que el autor explique en diversas ocasiones cómo se vinculan las propuestas actuales con otros estadios anteriores en el desarrollo de la teoría.

Como pequeña pega podría señalarse el hecho, advertido por el propio autor en el prólogo, de que entre los temas que no ha sido posible abordar en el libro por falta de espacio se encuentre la relación entre categorías funcionales y morfología, cuestión que consideramos especialmente relevante, dado que en ocasiones se propone que la morfología refleja superficialmente aspectos relativos a la estructura funcional de sintagmas u oraciones, o bien se recurre a datos que implican a la morfología flexiva para argumentar a favor de determinadas categorías funcionales. Así sucede, por ejemplo, cuando se discute la hipótesis propuesta por algunos autores de que el español expresaría género y número en el sustantivo con dos proyecciones funcionales diferentes, mientras que en italiano ambas categorías se expresarían en la misma proyección funcional (pp. 
123-124). Argumentos similares se encuentran en la página 130, cuando se debate si el aspecto está gramaticalizado en español como una categoría funcional, o en la página 131, cuando se afirma que el hecho de que la polaridad no se manifieste en la flexión verbal en español podría hacernos dudar de si se codifica como una categoría funcional en sintaxis. Es comprensible, sin embargo, la necesidad de acotar la materia tratada y, en ese sentido, creemos que la opción elegida por el autor - centrarse fundamentalmente en la sintaxis de las categorías funcionales y brevemente en su semántica, su adquisición y su pérdida- resulta coherente.

En suma, por todo lo dicho a lo largo de estas páginas, consideramos que Las categorías funcionales es un excelente manual de sintaxis generativista de nivel intermedio-avanzado, cuyo estudio recomendamos a todos aquellos estudiantes e investigadores que quieran conocer con cierta profundidad este modelo teórico.

\section{Bibliografía}

BOSQUE, I. (2015 [1989]): Las categorías gramaticales. Relaciones y diferencias. Madrid: Síntesis (2.ํe․).

CHOMsKY, N. (1995): The minimalist program. Cambridge (Mass.): MIT Press.

CINQUE, G. (1999): Adverbs and functional heads. Oxford: Oxford University Press.

GRIMSHAW, J. (1991): Extended projections. Manuscrito inédito. Brandeis University.

HARLEY, H. (1995): Subjects, events and licensing. Tesis doctoral, Cambridge (Mass.): MIT Press.

LARSON, R. (1988): “The double object construction”. Linguistic Inquiry 19, pp. 335-391.

RAmChAnd, G. (2008): First Phase Syntax. Cambridge: Cambridge University Press.

RodRíGuez RAmalle, T. (1995): "Sobre los adverbios demostrativos en español”. Cuadernos de Lingüística del Instituto Ortega y Gasset 3, 57-78.

WiLtschко, M. (2014): The universal structure of categories. Cambridge: Cambridge University Press. 